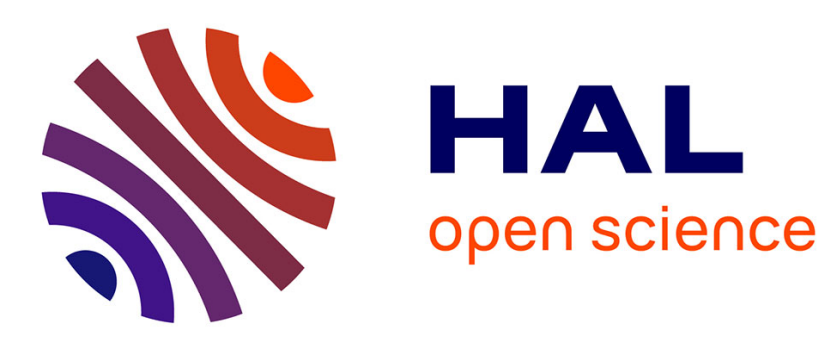

\title{
The resonant population of asteroids in librating states of the $\nu 6$ linear secular resonance
}

M Huaman, F. Roig, V Carruba, R C Domingos, Safwan Aljbaae

\section{To cite this version:}

M Huaman, F. Roig, V Carruba, R C Domingos, Safwan Aljbaae. The resonant population of asteroids in librating states of the $\nu 6$ linear secular resonance. Monthly Notices of the Royal Astronomical Society, 2018, 481 (2), pp.1707 - 1717. 10.1093/mnras/sty2381 . hal-02481210

\section{HAL Id: hal-02481210 https://hal.sorbonne-universite.fr/hal-02481210}

Submitted on 17 Feb 2020

HAL is a multi-disciplinary open access archive for the deposit and dissemination of scientific research documents, whether they are published or not. The documents may come from teaching and research institutions in France or abroad, or from public or private research centers.
L'archive ouverte pluridisciplinaire HAL, est destinée au dépôt et à la diffusion de documents scientifiques de niveau recherche, publiés ou non, émanant des établissements d'enseignement et de recherche français ou étrangers, des laboratoires publics ou privés. 


\title{
The resonant population of asteroids in librating states of the $v_{6}$ linear secular resonance
}

\author{
M. Huaman, ${ }^{1,2 \star}$ F. Roig, ${ }^{1}$ V. Carruba, ${ }^{2}$ R. C. Domingos ${ }^{3}$ and S. Aljbaae ${ }^{2}$ \\ ${ }^{1}$ Observatório Nacional (ON), Rua Gal. Jose Cristino 77, Rio de Janeiro RJ 20921-400, Brazil \\ ${ }^{2}$ São Paulo State University (UNESP), School of Natural Sciences and Engineering, Guaratinguetá SP 12516-410, Brazil \\ ${ }^{3}$ São Paulo State University (UNESP), Campus of São João da Boa Vista SP 13876-750, Brazil
}

Accepted 2018 August 30. Received 2018 August 27; in original form 2018 January 30

\begin{abstract}
We discuss the asteroid population evolving close by or into the $v_{6}$ secular resonance in the main asteroid belt. Using the analytical method of Yoshikawa, we estimate the actual domain of the $v_{6}$ resonance, which currently contains 4137 asteroids identified from the AstDys catalogue.We perform numerical integrations of the obits of these asteroids, considering the gravitational perturbation of all the planets, and identify those showing pure libration states. For the first time, seven asteroids are found to librate in aligned states, all located at the central main belt. We analyse the stability of this new population by extending our numerical integrations both into the past and into the future, to find that all of them are stable, but only four of them display long-term aligned librations. We also identified the asteroid families located close to and within the domain of the $v_{6}$ secular resonance, with a particular focus on the Svea family, which has 54 per cent of its members librating in anti-aligned states. This is the third family identified to be largely affected by the $v_{6}$ resonance, together with the families of Tina and Euphrosyne.
\end{abstract}

Key words: celestial mechanics - minor planets, asteroids: general.

\section{INTRODUCTION}

The $v_{5}, v_{6}$, and $v_{16}$ linear secular resonances in the asteroid belt occur when there is a one-to-one commensurability between the frequency of precession of an asteroid's perihelion, $g$, or the frequency of regression of an asteroid's node, $s$, and the corresponding frequencies of either Jupiter, $g_{5}$, or Saturn, $g_{6}, s_{6}$. The $v_{6}$ resonance, in particular, corresponds to the condition $g \approx g_{6}$. This resonance is of particular importance since it constitutes a major boundary for the distribution of asteroids in the main belt.

Tisserand (1882) and Charlier (1902) performed the first attempts to construct a secular theory of planetary motion using an expansion of the disturbing function to a high degree. Later on, Hagihara (1928) developed a high-order secular theory for Jupiter and Saturn, and Brouwer \& van Woerkom (1950) improved the linear secular theory for the outer planets to accurately determine the values of the fundamental frequencies $g_{5}, g_{6}, s_{6}$. In this context, Williams (1969) used a non-linear analytical theory to study the secular evolution of asteroids perturbed by the giant planets, and suggested that the linear secular resonances $v_{5}, v_{6}, v_{16}$ are strong mechanisms of asteroid depletion. Yoshikawa (1987), Knežević et al. (1991), Morbidelli \& Henrard (1991b), and Morbidelli (1993) studied in detail the dynamics and topology of the phase space of the linear secular resonances from the Hamiltonian approach.

The dynamics of the $v_{6}$ secular resonance is characterized by either the libration or the circulation of the resonant argument $\sigma_{6}=$ $\varpi-\varpi_{6}$, where $\varpi$ is the longitude of the asteroid perihelion and $\varpi_{6}$ is that of Saturn. In a circulating state, $\sigma_{6}$ varies continuously from $0^{\circ}$ to $360^{\circ}$, while in a librating state the resonant argument is bounded to oscillate around an equilibrium point. Two equilibria are possible, one around $0^{\circ}$ and another around $180^{\circ}$. The libration around $0^{\circ}$ is called an 'aligned libration', since in this configuration the two perihelia of the asteroid and the planet are roughly oriented in the same direction. On the other hand, the libration around $180^{\circ}$ is called an 'anti-aligned libration'; this latter libration usually occurs when the orbit of the asteroid is closer to the orbit of the planet (Yoshikawa 1987). Morbidelli \& Henrard (1991a) and Morbidelli (1993) described the $v_{6}$ resonance as an atypical case among secular resonances, because the locus of the resonance is almost independent on the orbital eccentricity (i.e. the resonance occurs at a given distance from the Sun and a given orbital inclination, but for all values of the eccentricity; Williams \& Faulkner 1981). Since a perihelion secular resonance pumps up the eccentricity while keeping the inclination approximately constant, a body evolving in the $v_{6}$ resonance may in principle have its eccentricity growing indefinitely. 

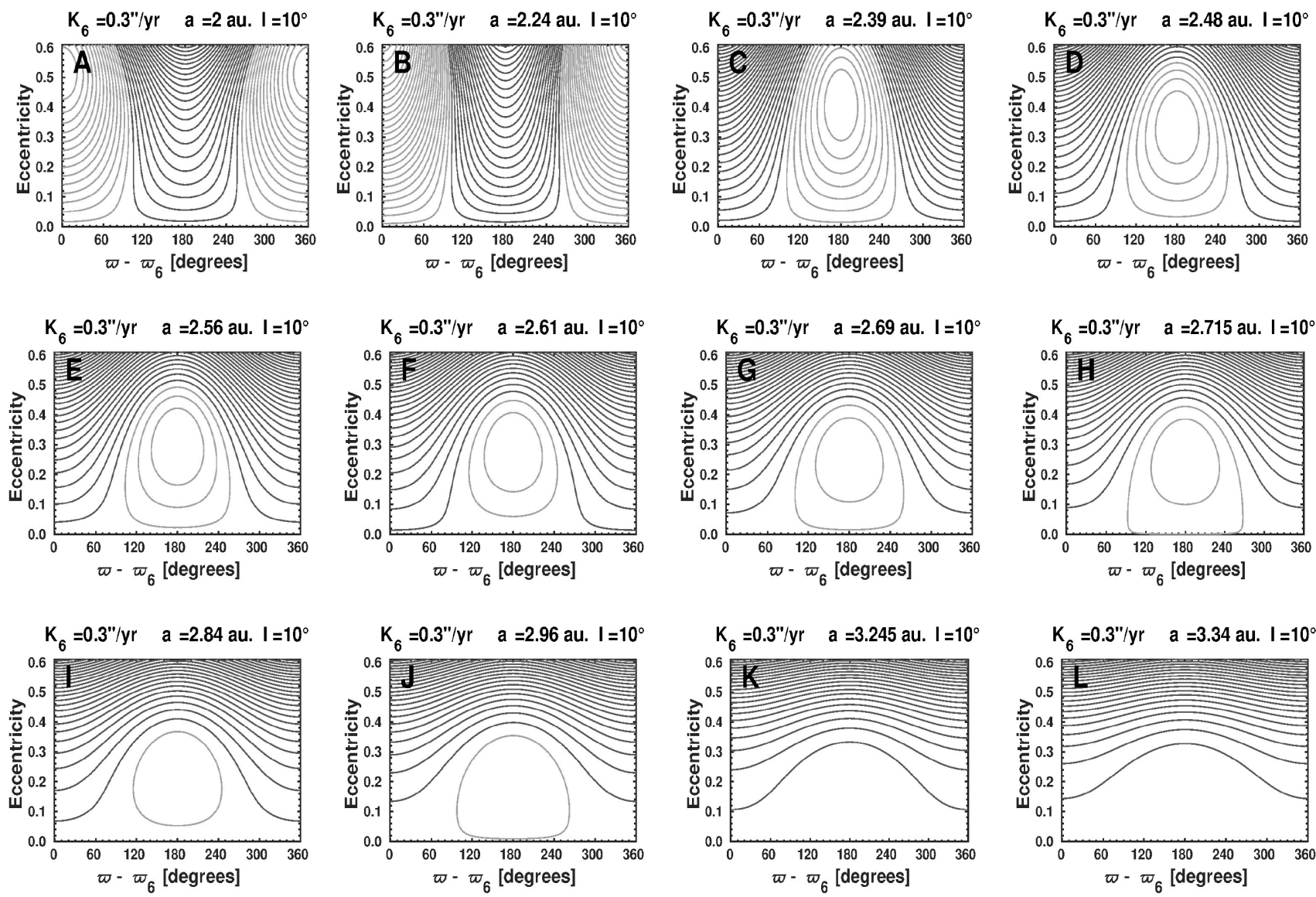

Figure 1. Level curves of the Hamiltonian model for the $v_{6}$ secular resonance according to Yoshikawa (1987) and Morbidelli \& Henrard (1991b) for $\sigma_{6}^{*} \rightarrow 0^{\circ}$ (equation 2). The parameter $k_{6}=0.3$ arcsec $\mathrm{yr}^{-1}$ corresponds to $b-g_{6}$ (see text). The upper row panels give the phase portraits for four different values of $a$ in the inner main belt. The middle row panels correspond to the central main belt, and the lower row panels to the outer main belt. Inclination is the same in all cases $\left(10^{\circ}\right)$. The dark and light grey colours identify the circulation and libration regimes, respectively. See text for discussion.

Numerical simulations by Froeschlé \& Scholl (1993) allowed the identification of several comets and asteroids showing antialigned librations within the $v_{6}$ resonance. The dynamical influence of this resonance on some asteroid families has been recently addressed by Carruba \& Morbidelli (2011a), who identified the family of Tina with all of its members in anti-aligned libration. Besides, Machuca \& Carruba (2012) and Carruba, Aljbaae \& Souami (2014) found 19 members of the family of Euphrosyne also in anti-aligned libration. These bodies may represent a transient population that is temporarily captured in the resonance, or they may be members of a long-lasting group of resonant bodies.

In this work, we propose to extend the previous results by performing a global study of the dynamical evolution of real asteroids that interact with the $v_{6}$ resonance. In Section 2, we apply the analytical method of Yoshikawa (1987) to estimate the possible boundaries of the resonance in terms of the orbital elements $a, e, I$ (semi-major axis, eccentricity, and inclination, respectively) and of the frequency $g$. In Section 3, we present the results of the numerical simulations that allow the identification of those asteroids within the resonance boundaries that show pure libration states. The effects of the $v_{6}$ resonance on the asteroid families are addressed in Section 4. Finally, Section 5 is devoted to the conclusions.

\section{TOPOLOGY AND DOMAIN OF THE $v_{6}$ SECULAR RESONANCE}

The analytical model of Yoshikawa (1987) considers that the dynamics of the $v_{6}$ resonance is approximately described by the following one degree of freedom Hamiltonian function:

$F_{v_{6}}=\frac{1}{2}\left(b-g_{6}\right) e^{2}+\frac{1}{4} c e^{4}-d e \cos \left(\varpi-\varpi_{6}^{*}\right)$

where $\varpi_{6}^{*}=g_{6} t+$ const., $b$ is a coefficient that depends on $a, I$, and $c, d$ are coefficients that depend only on $a$. The evolution of this Hamiltonian describes the changes in eccentricity and resonant argument, $\sigma_{6}^{*}=\varpi-\varpi_{6}^{*} \approx \sigma_{6}$, for different fixed values of the semi-major axis and inclination. The solutions of this Hamiltonian generate two different equilibrium points corresponding to the conditions $\mathrm{d} \sigma_{6}^{*} / \mathrm{d} t=0$ and $\mathrm{d} e / \mathrm{d} t=0$ : one equilibrium for $\sigma_{6}^{*}=0^{\circ}$ and the other for $\sigma_{6}^{*}=180^{\circ}$. Therefore, we can rewrite the Hamiltonian either as:

$F_{\nu_{6}}^{\prime}\left(\sigma_{6}^{*} \rightarrow 0^{\circ}\right)=\frac{1}{2}\left(b-g_{6}\right) e^{2}+\frac{1}{4} c e^{4}-d e \cos \left(\sigma_{6}^{*}\right)$

or

$F_{\nu_{6}}^{\prime}\left(\sigma_{6}^{*} \rightarrow 180^{\circ}\right)=\frac{1}{2}\left(b-g_{6}\right) e^{2}+\frac{1}{4} c e^{4}+d e \cos \left(\sigma_{6}^{* \prime}\right)$

where $\sigma_{6}^{* \prime}=\sigma_{6}^{*}+180^{\circ}$. According to Morbidelli \& Henrard (1991b), equation (2) gives the characteristic evolution of 

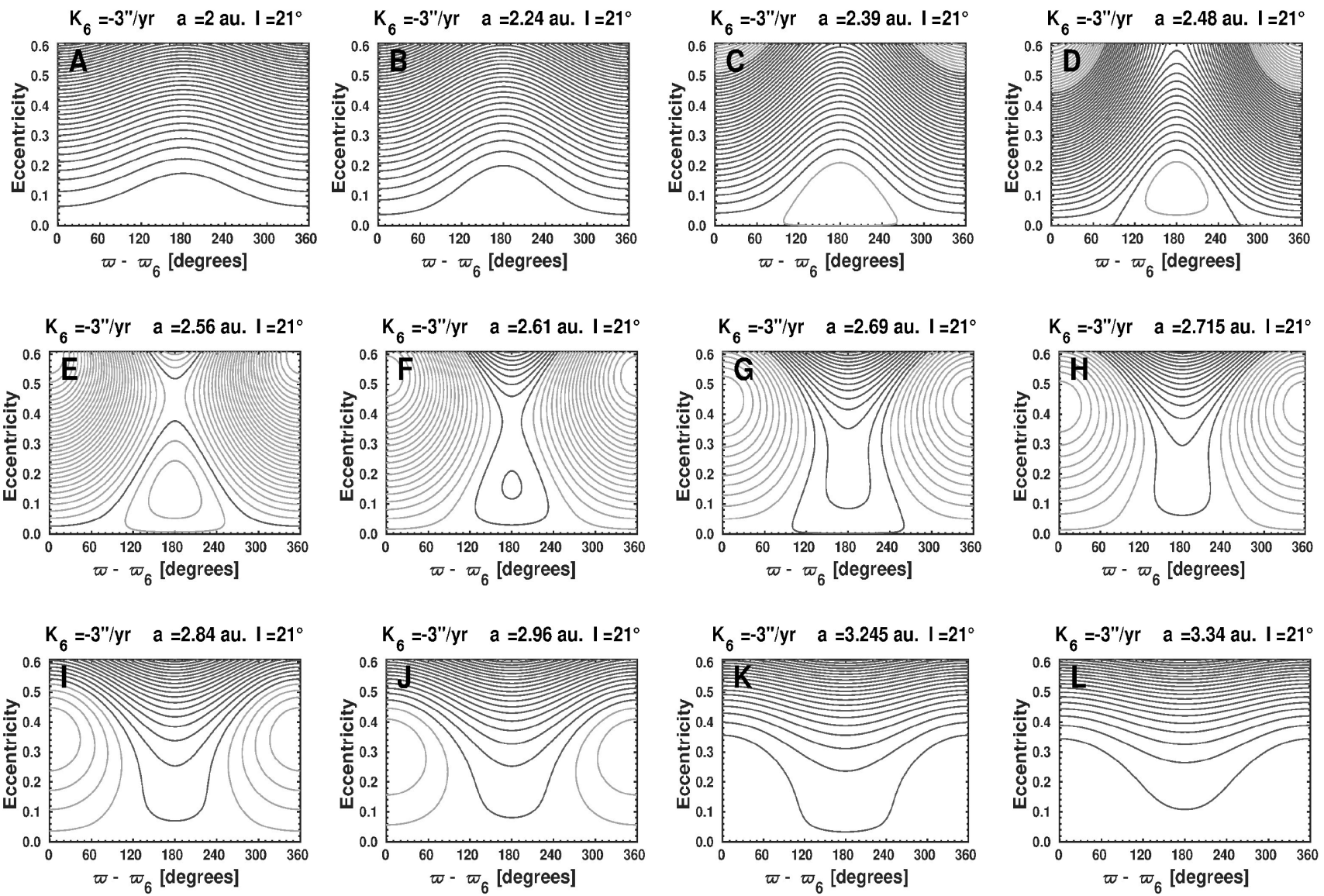

Figure 2. Same as Fig. 1, but for $\sigma_{6}^{*} \rightarrow 180^{\circ}$ (equation 3), $k_{6}=-3.0 \operatorname{arcsec}_{\mathrm{yr}}-1$, and inclination $21^{\circ}$.

Table 1. Ranges of the parameter $k_{6}=b-g_{6}$ of the Yoshikawa (1987) analytical model that lead to libration of the critical argument, in terms of the location in the main asteroid belt. The first column gives the location in the semi-major axis, the second column gives the interval of $k_{6}$ where librations can occur, and the third and fourth column discriminate between aligned and anti-aligned librations, respectively.

\begin{tabular}{llll}
\hline$a(\mathrm{au})$ & \multicolumn{3}{c}{$k_{6}$ interval $\left(\operatorname{arcsec}^{-1}\right)$} \\
& All librations & Aligned & Anti-aligned \\
\hline 2.00 & {$[-1,+1]$} & {$[-1,0]$} & +1 \\
2.24 & {$[-2,+2]$} & {$[-2,0]$} & {$[+1,+2]$} \\
2.39 & {$[-3,+3]$} & {$[-3,-2]$} & {$[0,+3]$} \\
2.48 & {$[-4,+3]$} & {$[-4,-2]$} & {$[-1,+3]$} \\
2.56 & {$[-5,+3]$} & {$[-5,-3]$} & {$[-3,+3]$} \\
2.66 & {$[-6,+3]$} & {$[-6,-4]$} & {$[-5,+3]$} \\
2.76 & {$[-6,+3]$} & {$[-6,-5]$} & {$[-6,+3]$} \\
2.86 & {$[-6,+3]$} & -6 & {$[-6,+3]$} \\
2.96 & {$[-6,+3]$} & & {$[-6,+3]$} \\
3.05 & {$[-6,+3]$} & & {$[-6,+3]$} \\
3.25 & {$[-6,+2]$} & & {$[-6,+3]$} \\
\hline
\end{tabular}

asteroids located at low inclinations $\left(I<20^{\circ}\right)$ and/or values of $a<$ $2.5 \mathrm{au}$. The dynamical behaviour of asteroids at high inclinations $(I$ $>20^{\circ}$ ), and/or values of $a>2.5 \mathrm{au}$, is represented by equation (3). Using these equations, it is possible to localize the resonant region where the libration states occur, and to identify asteroids that may be stuck there.
Figs 1 and 2 show the level curves in the plane $\left(\sigma_{6}, e\right)$ of the Hamiltonian equations (2) and (3), respectively. These curves are plotted for fixed values of the parameter $k_{6}=b-g_{6}$ and the inclination $I$. Values in the range $-6 \leq k_{6} \leq+6 \operatorname{arcsec} \mathrm{yr}^{-1}$ were selected to get the closest neighbourhood of the $v_{6}$ secular resonance. We varied the semi-major axis $a$ between 2.0 and 3.34 au to cover the classical main asteroid belt. At each region of the belt (inner: $2.0<$ $a<2.5$ au; middle or central: $2.5<a<2.82$ au; and outer: 2.82 $<a<3.3 \mathrm{au}$ ), we consider four different fixed values of $a$. These values of $a$ were chosen only to provide an example of how the libration state depends on the semi-major axis.

In Fig. 1, we find aligned librations only for values of eccentricity $e>0.5$ in the inner belt (panels A and B). The anti-aligned librations are present throughout most of the main belt, with decreasing eccentricities for larger semi-major axes. In the outer belt, we identify orbits circulating counterclockwise (panels $\mathrm{K}$ and L). In Fig. 2, the extrema of the main belt show orbits circulating counterclockwise (panels A, B) or clockwise (panels K, L), respectively. Aligned librations appear at high $e$ in the inner belt, but reach smaller eccentricities at the central and outer belt. On the other hand, anti-aligned librations appear at small eccentricities in the inner and central belt, and disappear in the outer belt. It is worth noting that the portraits shown in Figs 1 and 2 are specific for the indicated values of $k_{6}$, and have been chosen only as examples to give a global view of the resonant behaviour. The chosen values encompass the extrema of resonant motion, from solutions lying very close to the resonance centre $\left(\sim 0.3 \operatorname{arcsec}_{\mathrm{yr}^{-1}}\right)$ to solutions lying far from the resonance centre $\left(\sim 3 \operatorname{arcsec} \mathrm{yr}^{-1}\right)$. 

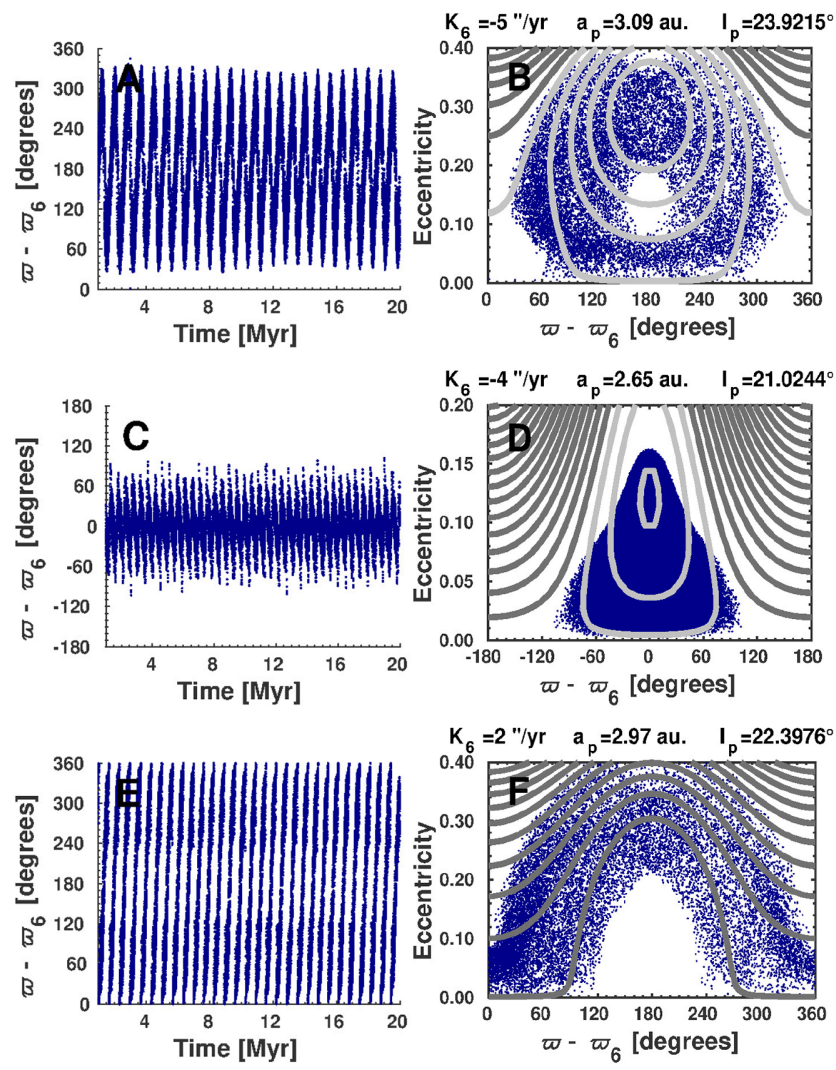

Figure 3. Temporal evolution of the resonant argument $\sigma_{6}=\varpi-\varpi_{6}$ of asteroids (322770) 2001 FD145 (panel A), (337335) 2001 EQ18 (panel C), and (154204) 2002 HL8 (panel E), exemplifying the three possible dynamical states related to the $v_{6}$ resonance: anti-aligned libration (around $180^{\circ}$ ), aligned libration (around $0^{\circ}$ ), and circulation, respectively. Panels B, $\mathrm{D}$, and $\mathrm{F}$ show the evolution in the plane $\left(\sigma_{6}, e\right)$ (blue dots), overlapped to the level curves of the analytical model for the corresponding value of $k_{6}$ (see text). Light grey levels identify the libration domains, while dark grey levels identify the circulation domain (note the different horizontal scale limits between panels $\mathrm{B}$ and D). The values of $a_{\mathrm{p}}$ and $I_{\mathrm{p}}$ are the synthetic proper elements of each asteroid, from AstDyS.

Other values of $k_{6}$ will produce different portraits. In Table 1 , we present a summary of the ranges in $k_{6}$ leading to resonant behaviour in terms of the semi-major axis, following our analysis of the Hamiltonian phase space (equations 2 and 3) throughout the main belt.

Our study of the analytical model allows us to conclude that the width of the libration region of main-belt asteroids in the $v_{6}$ resonance is, in general, $-6 \leq k_{6} \leq+3 \operatorname{arcsec} \mathrm{yr}^{-1}$. This analytical model, however, provides a limited contribution to address the actual dynamical evolution of resonant asteroids. In particular, the model is not accurate for large eccentricities. Nevertheless, we can use the above results to roughly estimate the boundaries of the aligned and anti-aligned libration regions, and apply this as a starting point to try to identify real asteroids trapped in these resonant states.

In order to better estimate the libration domain of the $v_{6}$ resonance, we define a libration parameter, $k_{\mathrm{Lib}}$, using the divisor $k_{\mathrm{Lib}}=g_{\mathrm{p}}-g_{6}$, where $g_{\mathrm{p}}$ is the proper frequency of the asteroid perihelion and $g_{6}$ is that of Saturn. Both frequencies, $g_{\mathrm{p}}$ and $g_{6}$, may be computed either from an analytical theory (e.g. Brouwer \& van Woerkom 1950; Milani \& Knežević 1990) or from a synthetic numerical theory (e.g. Nobili, Milani \& Carpino 1989; Knežević \& Milani 2003), and each theory may give different values of $k_{\mathrm{Lib}}$, even for the same asteroid. In our case, the value of $k_{\mathrm{Lib}}$ is only taken as a reference to select resonant candidates, and we assume that resonant motion may be constrained by the condition $-3.5 \leq k_{\mathrm{Lib}} \leq+3.0 \operatorname{arcsec}_{\mathrm{yr}}{ }^{-1}$. This constraint is based on our previous knowledge of the libration domain provided by the parameter $k_{6}$ of the Yoshikawa (1987) model (cf. Table 1). It is worth noting, however, that $k_{\mathrm{Lib}}$ is equivalent to but not the same as $k_{6}$, because they are obtained from essentially different approaches. Nevertheless, using $k_{6}$ as a proxy, we expect that our assumption for $k_{\mathrm{Lib}}$ encompasses a wide region where asteroids could be in libration states. We recall that other authors assume narrower values, like Froeschlé et al. (1995) $\left(0.35 \leq k_{\mathrm{Lib}} \leq 1.5 \operatorname{arcsec}_{\mathrm{yr}}{ }^{-1}\right)$ or Carruba (2009) $\left(-2.55 \leq k_{\mathrm{Lib}} \leq 2.55 \operatorname{arcsec}^{-1}\right)$.

Here we use two different sets of proper elements $a_{\mathrm{p}}, e_{\mathrm{p}}, \sin I_{\mathrm{p}}$, and frequencies $g_{\mathrm{p}}$ to compute the values of $k_{\mathrm{Lib}}$. The first set is given by the synthetic theory of Knežević \& Milani (2003), and it is listed in the AstDys catalogue of synthetic proper elements (version of 2017 April 16; available at hamilton.dm.unipi.it/astdys/). The main advantage of this catalogue is that it provides the values of the proper frequencies for a very large set of asteroids, with especially accurate values for high-eccentricity and high-inclination orbits (which is, in general, the main limitation of the analytical theories). The second set of proper elements/frequencies is computed by ourselves for a limited number of asteroids, from a digital filtering of the series $e$ $\cos \varpi, e \sin \varpi$, as explained in the next section.

\section{DYNAMICS OF ASTEROIDS INTERACTING WITH THE $v_{6}$ RESONANCE}

Taking into account the resonant domain defined in the previous section, we identify 4137 asteroids from the AstDys catalogue that might interact with the $v_{6}$ resonance in libration states according to their values of $k_{\mathrm{Lib}}$. This candidate population spans a range in $2.0107 \leq a_{\mathrm{p}} \leq 3.276 \mathrm{au}, 0.010 \leq e_{\mathrm{p}} \leq 0.434,0.435^{\circ} \leq I_{\mathrm{p}} \leq 33.322^{\circ}$, and absolute magnitude $H<20$. In principle, only some of these asteroids are expected to stay in pure libration states, while most of them are expected to interact with the $v_{6}$ resonance partially, by alternating between libration and circulation states.

In order to find the pure librators, we perform a numerical simulation of the orbital evolution of these 4137 candidates using the symplectic $N$-body integrator SWIFT_MVS (Levison \& Duncan 1994), including the modifications by Brož (1999). Our model takes into account the direct perturbation of the planets from Venus to Neptune, and the indirect perturbation of Mercury through the corresponding barycentric correction to the position and velocity of the Sun. The simulations span $20 \mathrm{Myr}$, with a time-step of 2 days. Such a time-span is long enough to detect the long-term effects of secular resonances (Carruba \& Machuca 2011b), and to identify the orbits that are presently locked inside the $v_{6}$ resonance. Examples of the behaviours identified by this procedure are shown in Fig. 3 for asteroids (322770) 2001 FD145 (anti-aligned libration), (337335) 2001 EQ18 (aligned libration), and (154204) 2002 HL8 (circulation). In the right-hand panels of Fig. 3, we overlap the dynamical evolution of the orbits in the $\left(\sigma_{6}, e\right)$ phase space to the Hamiltonian level curves of the analytical model (Section 2).

We found that 803 asteroids display pure librations (aligned and anti-aligned) around the equilibria of the $v_{6}$ resonance. Most of these asteroids have small proper eccentricities, in general less than 0.15 . We also found that 545 asteroids were removed from the main belt before $20 \mathrm{Myr}$ by planetary close encounters. Of these, 413 displayed temporary librations (either aligned, anti-aligned, or both) alternated with circulations, before being ejected. We do not 

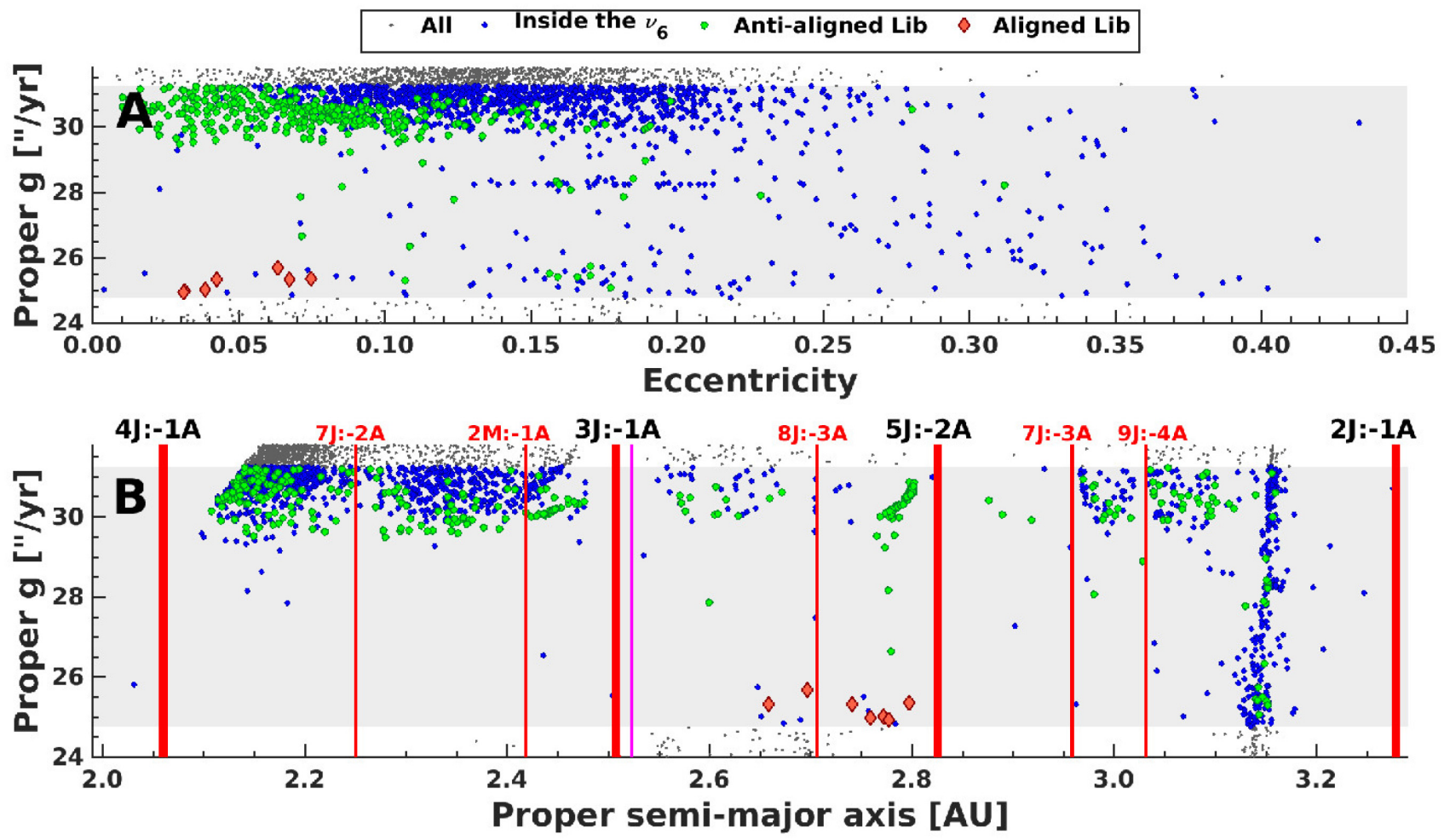

Figure 4. Distribution of asteroids around the $v_{6}$ secular resonance, projected onto the planes $\left(g_{\mathrm{p}}, e_{\mathrm{p}}\right)$ (panel A) and $\left(g_{\mathrm{p}}, a_{\mathrm{p}}\right)$ (panel B). The proper elements and frequency come from AstDyS (Knežević \& Milani 2003). The grey area represents the resonance domain. Dark grey dots are main-belt asteroids that are unlikely to be affected by the resonance. Green circles represent anti-aligned librators, red diamonds represent aligned librators, and blue circles either are circulators or alternate between libration and circulation. The classification is based on a 20 Myr numerical simulation of the orbital evolution. Vertical red lines in panel B give the approximate location of the main mean motion resonances with Jupiter and Mars. The vertical alignment of orbits observed at about 3.1 au in panel B is related to forced oscillations induced by the nearby 2:1 mean motion resonance with Jupiter.

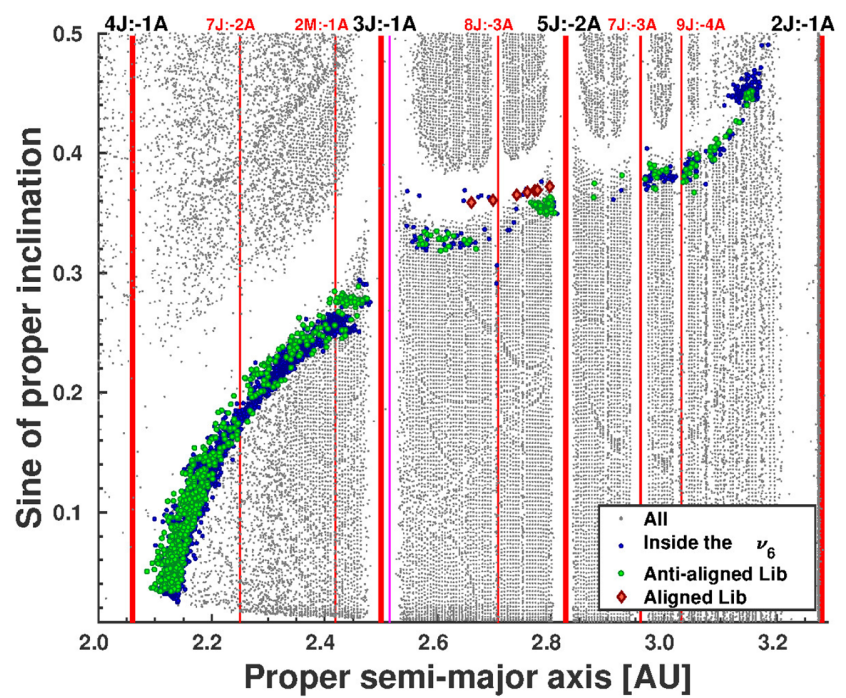

Figure 5. Distribution of the 4137 asteroids (coloured symbols) that interact with the $v_{6}$ resonance. The colour code is the same in Fig. 4. Dark grey dots represent proper elements of an initially uniform grid of test orbits (see text for explanation). Vertical red lines indicate the location of the main mean motion resonances.

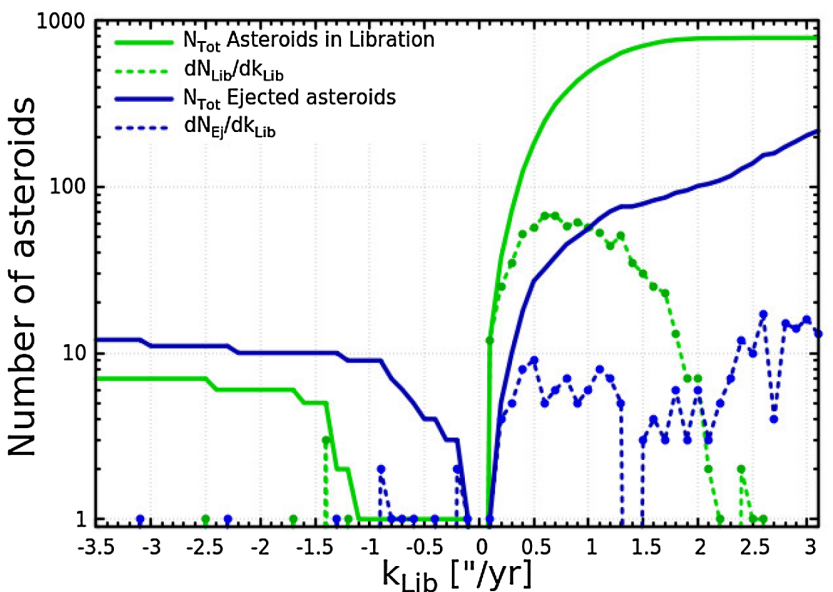

Figure 6. Distributions in terms of $k_{\mathrm{Lib}}$ of the number of asteroids that are in pure libration states, both aligned and anti-aligned, over $20 \mathrm{Myr}$ of evolution (803 asteroids, in full green line), and those asteroids that escape due to planetary encounters over the same time-span (545 asteroids, in full blue line). Dashed lines with dots are the differential distributions at bins of $0.1 \operatorname{arcsec} \mathrm{yr}^{-1}$. Full lines are the cumulative distributions. 
find any preference for the ejection to happen when the orbit was librating or circulating.

In Fig. 4, we show the distribution of the real asteroids (with symbols) that lie close to or inside the $v_{6}$ secular resonance, projected onto the planes $\left(e_{\mathrm{p}}, g_{\mathrm{p}}\right)$ (panel A) and $\left(a_{\mathrm{p}}, g_{\mathrm{p}}\right)$ (panel B). The assumed domain of the resonance $\left(g_{6} \pm k_{\mathrm{Lib}}\right)$ is indicated by the grey area; the 4137 candidates analysed in this study lie within this region. Green circles represent the orbits in pure anti-aligned libration (796 asteroids), while red diamonds are those in pure aligned libration (7 asteroids). Blue circles (3 334 asteroids) display either pure circulation or alternate between libration and circulation. Strong depletion is observed at the locations of the main mean motion resonances with Jupiter (vertical lines in panel B), as expected. While the anti-aligned librators appear to be spread throughout the whole asteroid belt (with a concentration at the inner belt), with values of $k_{\mathrm{Lib}}$ mostly positive, the aligned librators appear restricted to the central belt, with negative values of $k_{\mathrm{Lib}}$, in good agreement with the predictions of the analytical model (see Section 3.1).

Interestingly, both populations of pure librators (aligned and antialigned) are found close to the edges of the resonant domain, giving the impression that libration amplitudes of $\sigma_{6}$ tend to be large. The central part of the resonant domain, close to the equilibria ( $g_{\mathrm{p}} \simeq g_{6}$ ), is empty. This seems to be an artefact of using the AstDys synthetic proper elements, which are not suitable to describe the distribution of asteroids locked in librating states (e.g. Milani et al. 2017). Actually, 80.7 percent of the 4137 asteroids in our sample show errors in the proper eccentricity $\sigma(e)>0.01$, while 48,2 per cent show $\sigma(e)>0.02$, and 25 per cent show $\sigma(e)>0.03$. ${ }^{1}$ The errors in $a_{\mathrm{p}}$ and $\sin I_{\mathrm{p}}$, on the other hand, are almost negligible [0.01 per cent with $\sigma(a)>0.01$, and 0.16 per cent with $\sigma(\sin I)>$ $0.01]$. Such significant errors in $e_{\mathrm{p}}$ are produced by the proximity of the orbits to the strong $v_{6}$ secular resonance. As pointed out by Milani et al. (2017), this happens because the standard computation of the synthetic proper elements applied to resonant orbits is affected by the presence of resonant modes that generate large oscillations of the resulting proper elements. Therefore, to overcome this limitation, it is necessary to compute proper elements and frequencies specifically tailored for the $v_{6}$ resonance.

Here we recompute the synthetic proper elements/frequencies for the 803 asteroids in librating states following the approach described in Carruba (2010). This approach applies the frequency-modified Fourier transform method of Sidlichovský \& Nervorný (1996) to filter the series $e \cos \varpi$, esin $\varpi$. We consider a time-span of this series of $2 \mathrm{Myr}$, and apply a two-stage filter to obtain values of the proper element/frequency every $150 \mathrm{kyr}$. Then we compute the average proper element/frequency and their corresponding $\sigma$ dispersions over the whole time-span. These proper elements and frequencies differ from those provided by the AstDys catalogue, and they display much smaller $\sigma$ dispersions. Thus, they are expected to be more suitable to describe the distribution of asteroids close to the $v_{6}$ resonance.

In Fig. 5, we plot the distribution of 4137 asteroids that interact with the $v_{6}$ resonance projected onto the $\left(a_{\mathrm{p}}, \sin I_{\mathrm{p}}\right)$ plane. The distribution is overlapped by a dynamical map of the whole main belt (dark grey dots) obtained by computing synthetic proper elements for a grid of orbits, as explained above. The grid is constituted of $220 \times 350=77000$ test particles with osculating initial conditions

\footnotetext{
${ }^{1}$ The error $\sigma(e)$ is given in the AstDys catalogue, and it is a measure of the stability of the computed proper element, i.e. how much the proper element is expected to change over a long time-span.
}
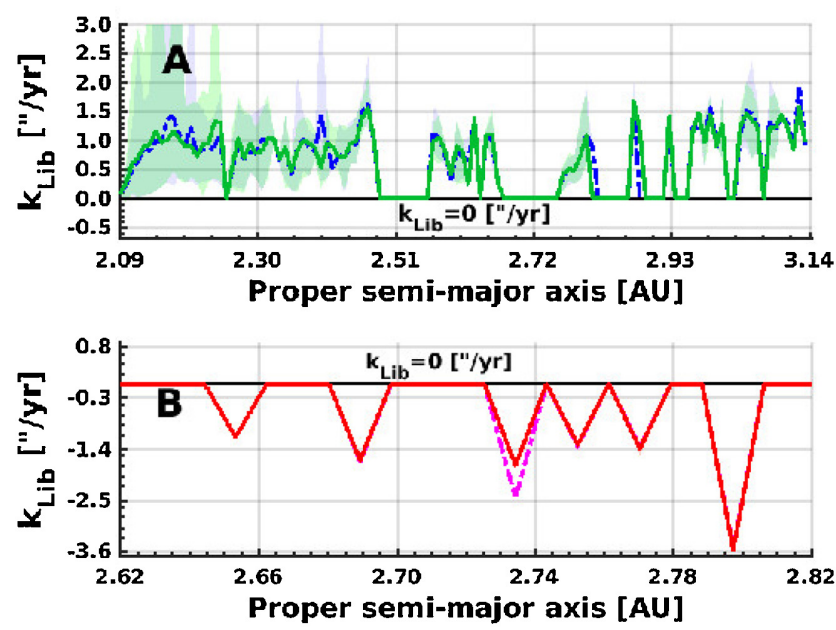

Figure 7. Distribution of values of $k_{\mathrm{Lib}}$ with respect to $a_{\mathrm{p}}$ for the antialigned (panel A) and the aligned (panel B) pure librators, respectively. The distributions are binned every 0.005 au. Full lines represent the mean value at each bin; the green area in panel A spans the maximum and minimum values at each bin. No similar area is plotted in panel B since the distribution of aligned librators is limited to six asteroids. The dashed blue and magenta lines give the distributions in terms of the present proper frequency, while the full green and red lines give the distribution in terms of the frequency computed after $20 \mathrm{Myr}$ of evolution.

distributed uniformly in the $(a, I)$ plane, with a spacing of 0.005 au and $0.2^{\circ}$. The initial values of the remaining osculating orbital elements $e, \varpi, \Omega$ (longitude of node), and $\lambda$ (mean longitude) of each test particle of the grid have been arbitrarily fixed to those of asteroid (410) Chloris, an asteroid located in the central main belt. Therefore, the dynamical map is expected to represent a slice of the proper elements space at constant $e_{\mathrm{p}} \simeq 0.26$. Both the grid of initial conditions and the 4137 asteroids are evolved using the symplectic integrator SWIFT_MVS over $20 \mathrm{Myr}$, and accounting for the gravitational perturbations of all the planets as previously described. It is worth noting that the synthetic proper elements for the real asteroids and the grid test particles are calculated from this evolution during the last $2 \mathrm{Myr}$, i.e. between 18 and $20 \mathrm{Myr}$.

Forty-five percent of the initial grid is depleted during the simulation, so the dynamical map in Fig. 5 gives the distribution of the surviving 42300 orbits. As expected, the $v_{6}$ resonance opens a wide gap in the grid all across the main belt, providing a barrier that shapes the upper boundary in the inclination of the asteroid belt. The large depletion of test particles observed around $\sim 2$ au is related to the overlap of the $v_{6}$ resonance with other resonances, in particular the $v_{16}$. We note that the aligned librators (red diamonds) are located at higher inclinations than the anti-aligned librators (green circles) in the same region of the main belt. This is related to the fact that the former have smaller values of $g_{\mathrm{p}}\left(k_{\mathrm{Lib}}<0\right)$ than the latter (compare to Fig. 4).

Once we have identified the 803 asteroids currently in pure libration states (both aligned and anti-aligned), and we have recalculated their proper elements and frequencies, we may use them to reassess the range of $k_{\mathrm{Lib}}$ that characterizes the resonant motion. In Fig. 6, we show the differential (dashed green line) and cumulative (full green line) distributions of the number of librating asteroids as a function of $k_{\mathrm{Lib}}$. In this case, $k_{\mathrm{Lib}}$ was calculated using the 'current' proper frequency, i.e. the proper frequency computed over the first 2 Myr of evolution. The figure also displays the differential and cumulative distributions (in full blue line) of the 545 asteroids that 
Table 2. List of asteroids evolving in either pure aligned libration or alternating between aligned libration and circulation. The second column gives the absolute magnitude; the third to sixth columns give the values of the synthetic proper elements and perihelion frequency; the last two columns are the backward and forward lifetimes of the orbits, indicating when applicable whether the orbit is scattered to the inner $(\mathrm{I})$ or to the outer $(\mathrm{O})$ Solar System. A $(*)$ symbol identifies the orbits that remain in pure aligned libration over $400 \mathrm{Myr}$ of evolution.

\begin{tabular}{|c|c|c|c|c|c|c|c|}
\hline Asteroid number & $H$ & $\begin{array}{l}a_{\mathrm{p}} \\
(\mathrm{au})\end{array}$ & $e_{\mathrm{p}}$ & $\sin I_{\mathrm{p}}$ & $\begin{array}{c}g_{\mathrm{p}} \\
\left(\operatorname{arcsec} \mathrm{yr}^{-1}\right)\end{array}$ & $\begin{array}{l}T_{\text {surv }}^{-} \\
(\mathrm{Myr})\end{array}$ & $\begin{array}{c}T_{\text {surv }}^{+} \\
(\mathrm{Myr})\end{array}$ \\
\hline \multicolumn{8}{|c|}{ Currently in pure libration } \\
\hline $337335(*)$ & 15.26 & 2.6583 & 0.04246 & 0.3587 & 25.3124 & 200.0 & 200.0 \\
\hline 143199 & 15.05 & 2.6971 & 0.06351 & 0.3604 & 25.6695 & 200.0 & 200.0 \\
\hline 295883 & 15.45 & 2.7413 & 0.06737 & 0.3650 & 25.3128 & 200.0 & 200.0 \\
\hline $73415(*)$ & 14.76 & 2.7591 & 0.03151 & 0.3671 & 24.9691 & 200.0 & 200.0 \\
\hline $322878(*)$ & 15.76 & 2.7718 & 0.03859 & 0.3680 & 25.0071 & 200.0 & 200.0 \\
\hline $371246(*)$ & 15.68 & 2.7771 & 0.03122 & 0.3688 & 24.9152 & 200.0 & 200.0 \\
\hline 203236 & 14.63 & 2.7978 & 0.07478 & 0.3718 & 25.3434 & 200.0 & 200.0 \\
\hline \multicolumn{8}{|c|}{ Currently alternating } \\
\hline 397488 & 16.84 & 2.5453 & 0.03481 & 0.3689 & 24.8544 & 14.1 (I) & 27.6 (I) \\
\hline 248158 & 15.61 & 2.6732 & 0.06833 & 0.3628 & 24.8347 & 163.1 & 200.0 \\
\hline 401320 & 16.74 & 2.6765 & 0.09075 & 0.3638 & 24.8219 & 129.5 & 200.0 \\
\hline 354463 & 15.91 & 2.6901 & 0.04604 & 0.3629 & 24.9176 & 200.0 & 200.0 \\
\hline 364329 & 16.84 & 2.6948 & 0.07527 & 0.3624 & 25.2392 & 200.0 & 200.0 \\
\hline 314787 & 16.11 & 2.7511 & 0.06212 & 0.3658 & 25.2298 & 200.0 & 200.0 \\
\hline 257972 & 15.07 & 3.1245 & 0.21953 & 0.4399 & 25.2417 & $18.3(\mathrm{O})$ & $6.3(\mathrm{O})$ \\
\hline 179554 & 14.70 & 3.1282 & 0.23968 & 0.4437 & 25.1906 & 13.6 & 114.1 \\
\hline 88064 & 13.43 & 3.1289 & 0.28837 & 0.4496 & 25.0522 & $12.9(\mathrm{O})$ & 80.2 \\
\hline 246505 & 14.52 & 3.1362 & 0.29986 & 0.4529 & 25.7276 & $115.7(\mathrm{O})$ & $8.8(\mathrm{O})$ \\
\hline 320989 & 15.20 & 3.1378 & 0.32547 & 0.4572 & 25.5468 & $131.1(\mathrm{O})$ & $9.8(\mathrm{O})$ \\
\hline 282444 & 14.73 & 3.1384 & 0.19053 & 0.4474 & 24.9942 & 54.4 & 52.7 \\
\hline 220835 & 14.73 & 3.1391 & 0.20395 & 0.4494 & 24.9588 & $19.6(\mathrm{O})$ & $16.5(\mathrm{O})$ \\
\hline 121759 & 13.91 & 3.1403 & 0.17451 & 0.4463 & 25.6889 & 200.0 & 49.1 \\
\hline 56932 & 13.51 & 3.1427 & 0.16097 & 0.4473 & 26.1453 & 200.0 & 15.7 \\
\hline 313169 & 14.53 & 3.1464 & 0.29678 & 0.4585 & 26.7213 & $17.4(\mathrm{O})$ & $18.0(\mathrm{O})$ \\
\hline 344837 & 15.08 & 3.1522 & 0.21230 & 0.4527 & 28.2298 & 200.0 & $67.5(\mathrm{O})$ \\
\hline 267506 & 14.79 & 3.1522 & 0.27848 & 0.4504 & 31.1599 & $8.4(\mathrm{O})$ & $21.8(\mathrm{O})$ \\
\hline 406770 & 15.28 & 3.1530 & 0.26629 & 0.4525 & 30.4131 & 56.3 & $55.1(\mathrm{O})$ \\
\hline 165949 & 14.51 & 3.1533 & 0.35400 & 0.4742 & 25.1702 & $56.2(\mathrm{O})$ & $16.6(\mathrm{O})$ \\
\hline 402513 & 15.07 & 3.1537 & 0.29342 & 0.4547 & 30.6264 & 200.0 & $102.4(\mathrm{I})$ \\
\hline
\end{tabular}

were removed from the main belt by planetary close encounters. We note that the distribution of anti-aligned resonant orbits shows values of $k_{\mathrm{Lib}}>0$, as expected, with a maximum at $0.6 \operatorname{arcsec} \mathrm{yr}^{-1}$. The corresponding cumulative distribution saturates for values of $k_{\text {Lib }}>2.5 \operatorname{arcsec}^{-1}$. On the other hand, the distribution of aligned resonant orbits shows values of $k_{\mathrm{Lib}}<0$, and the cumulative distribution saturates for $k_{\mathrm{Lib}}<-2.5 \operatorname{arcsec}_{\mathrm{yr}}{ }^{-1}$. These saturation limits better define the criterion for resonant motion to arise. Concerning the escaping orbits, we note an increasing number of them for $k_{\mathrm{Lib}}>2 \operatorname{arcsec} \mathrm{yr}^{-1}$, which is related to the fact that the sample of 4137 candidates is concentrated at positive values of $k_{\mathrm{Lib}}$, especially in the inner belt.

Fig. 7 shows the distribution of values of $k_{\mathrm{Lib}}$ in terms of the proper semi-major axis for the 803 asteroids in pure libration states. The distribution of the population is given in terms of the 'current' proper frequencies, i.e. the proper frequency computed over the first 2 Myr of evolution (dashed blue and magenta lines for anti-aligned and aligned libration, respectively), and also in terms of the proper frequencies computed over the last $2 \mathrm{Myr}$ of evolution (full green and red lines for anti-aligned and aligned libration, respectively). These latter correspond to the proper elements shown in Fig. 5. The full and dashed lines represent the mean value of $k_{\mathrm{Lib}}$ at each bin of $0.005 \mathrm{au}$. For the anti-aligned librators (panel A), the green shaded area encompasses the maximum and minimum values of $k_{\text {Lib }}$ at each bin. No significant differences are appreciated between the current frequency and the frequency after 20 Myr. Fig. 7 contrasts with the distribution shown in Fig. 4, confirming that the frequencies we compute seem to be much more suitable to describe the secular resonant motion than those of AstDys, as previously mentioned.

\subsection{The aligned librators}

Asteroids in anti-aligned libration of the $v_{6}$ resonance have been previously identified by other authors, but asteroids in aligned libration are actually a new finding. The sparse number of such bodies, and in particular the lack of them in the inner belt, seems to be related to the significant depletion observed in the regions of the main belt where they are likely to be found. The analytical model of Yoshikawa (1987) shows that aligned librators must always have small eccentricities, and should be found for values of $-3.0 \leq k_{6} \leq 1.0 \operatorname{arcsec} \mathrm{yr}^{-1}$ in the inner belt and values of $k_{6} \leq-2.0 \operatorname{arcsec}^{-1}$ in the central and outer belt. 


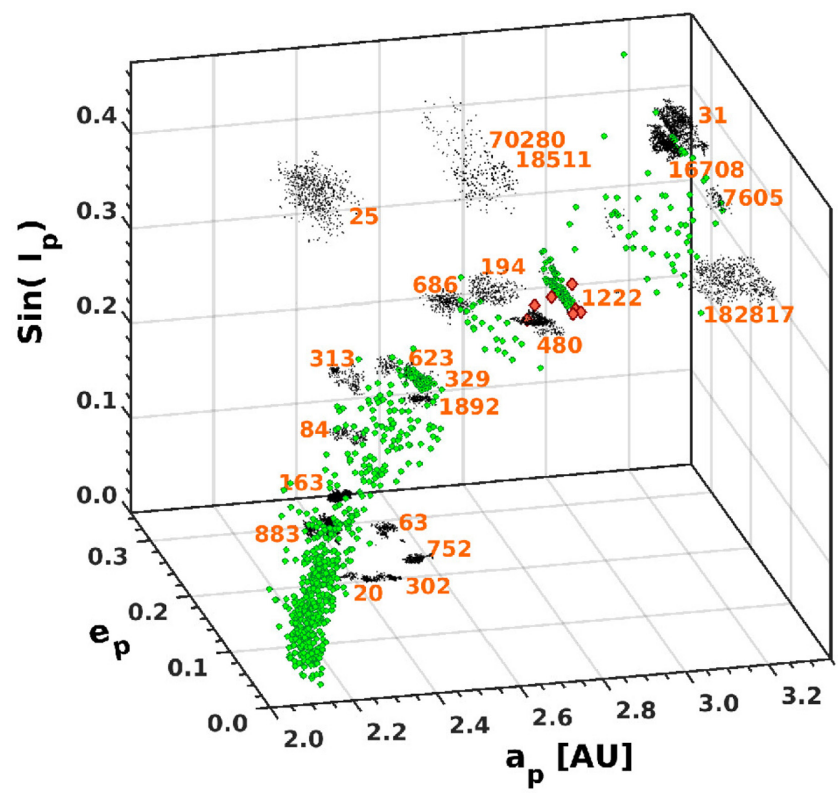

Figure 8. Distribution in the 3D space of proper elements of the 25 asteroid families (clusters of black dots) considered in this study. The population of the $v_{6}$ pure librators is also shown (green circles and red diamonds for antialigned and aligned, respectively). To plot the families, we take into account only asteroids with absolute magnitude $H<16$. Numbers correspond to the numbers of the asteroids that give the name to each family. The pure librators are displayed in terms of the proper elements computed in Section 3 (Fig. 5). The families are displayed in terms of the AstDys proper elements (Knežević \& Milani 2003).

In this study, we identify for the first time 7 asteroids currently in aligned libration, all in the central main belt with values of $k_{\mathrm{Lib}}$ ranging from -3.3 to $-1.2 \operatorname{arcsec}^{-1}$. Asteroid (337335) 2001 EQ18, whose evolution is shown in Fig. 3, is one of them. It is worth noting that this classification is based on the orbital behaviour over a 20 Myr time-span only. Besides, we identified 21 other asteroids in the central and outer main belt that alternate between aligned libration and circulation over $20 \mathrm{Myr}$. These asteroids are reported in Table 2.

We analysed the long-term orbital stability of this population of 28 asteroids by extending our numerical simulations to $200 \mathrm{Myr}$ into the future and $200 \mathrm{Myr}$ into the past. The initial conditions for the backwards simulations were set up by changing the sign of the Cartesian velocity components, following the procedure described in Carruba, Nesvorný \& Vokrouhlický (2016). In order to track the orbits backwards with enough precision, we set the time-step of these simulations to 1 day. The results show that only 4 of the 7 asteroids classified as pure librators remained in stable orbits with the resonant argument $\sigma_{6}$ always librating around $0^{\circ}$ for the whole 400 Myr time-span. These are indicated in Table 2 by the symbol $(*)$. The other 3 pure librators are affected by the nearby $8: 3$ mean motion resonance with Jupiter, and start to alternate between libration and circulation. The asteroids that survive in stable orbits for the whole time-span, with the resonance argument alternating between libration (aligned and/or anti-aligned) and circulation, are observed in the region of semi-major axis $2.69<a_{\mathrm{p}}<2.75$ au and eccentricity less than 0.08 .

The remaining asteroids, i.e. those that do not survive over the 400 Myr time-span, represent more than half of the asteroids that alternate between libration and circulation of the resonant argument, and are scattered into the outer belt (with semi-major axis $3.12<$ $\left.a_{\mathrm{p}}<3.154\right)$. They are removed by planetary close encounters, in most cases with Jupiter. The lifetimes of these orbits are reported in the last two columns of Table 2.

\section{ASTEROID FAMILIES INTERACTING WITH THE $v_{6}$ RESONANCE}

Asteroid families are groups of asteroids that have a common origin from the collisional fragmentation of a larger parent body. Identifying an asteroid family that may interact with a secular resonance is relevant, since the dynamical lifetimes of the resonant family members may help to constrain the age of the family.

Here, we consider a list of 195 known asteroid families and clumps in the main asteroid belt that have been reported by different authors (Mothe-Diniz, Roig \& Carvano 2005; Gil-Hutton 2006; Carruba \& Machuca 2011b; Machuca \& Carruba 2012; Carruba et al. 2014; Milani et al. 2014; Nesvorný, Brož \& Carruba 2015; Spoto, Milani \& Knežević 2015; Milani et al. 2016), and from this list, we select 25 families that are located in the neighbourhood of the $v_{6}$ resonance domain. The distribution of these families is shown in Fig. 8 (black dots), together with the population of aligned (red diamonds) and anti-aligned (green circles) librators. Among the selected families, there are the families of Tina and Euphrosyne, which have been previously identified as interacting with the $v_{6}$ resonance. Besides, we identify four other families that also appear to partially interact with this resonance: Lucienne, Svea, Cindygraber, and 1995 SP1. We will focus, hereafter, on the first two.

Since an asteroid family forms a cluster in the space of proper elements, a usual method to detect families is the hierarchical clustering method (Zappalà et al. 1990). This consists in defining a distance metric in terms of proper elements, $d\left(a_{\mathrm{p}}, e_{\mathrm{p}}, \sin I_{\mathrm{p}}\right)$, and to group together pairs of asteroids for which the mutual distance is less than a given cut-off value. The form of the metric is related to the shape of the ejection velocity field during the formation of the family, and the cut-off distance is the expected maximum ejection velocity, $v_{\text {cut }}$. Therefore, the larger the $v_{\text {cut }}$, the larger the number of asteroids that become members of a given family. ${ }^{2}$

Fig. 9 shows the number of members of the Lucienne (panel A) and Svea (panel B) families as a function of the cut-off velocity. The blue line is the cumulative number, while the cyan line is the differential distribution. In order to determine the minimum and maximum possible number of family members, we take into account the peaks in the differential distribution. For example, the family of Lucienne starts with 37 members at $v_{\text {cut }}=37 \mathrm{~m} \mathrm{~s}^{-1}$ and reaches a maximum of 254 members at $v_{\text {cut }}=152 \mathrm{~m} \mathrm{~s}^{-1}$. For larger cut-off values, the family slowly expands including peripheral objects that might not be true members of the family: the so-called halo of the family.

In Fig. 10, we show the distribution of the Lucienne (circles) and Svea (diamonds) family members projected onto the $\left(a_{\mathrm{p}}, \sin I_{\mathrm{p}}\right)$ plane (panel A) and the $\left(g_{\mathrm{p}}, \sin I_{\mathrm{p}}\right)$ plane (panel B). Both families are located in the inner main belt, in a narrow region between the 2:1 mean motion resonance with Mars and the 3:1 mean motion

${ }^{2}$ We use the standard metric

$d=n a_{\mathrm{p}} \sqrt{\frac{5}{4}\left(\frac{\delta a_{\mathrm{p}}}{a_{\mathrm{p}}}\right)^{2}+2\left(\delta e_{\mathrm{p}}\right)^{2}+2\left(\delta \sin I_{\mathrm{p}}\right)^{2}}$

where $n$ is the orbital mean motion (so $d$ has units of $\left.\mathrm{m} \mathrm{s}^{-1}\right)$, and $\left(\delta a_{\mathrm{p}}, \delta e_{\mathrm{p}}\right.$, $\delta \sin I_{\mathrm{p}}$ ) are the mutual separations. 

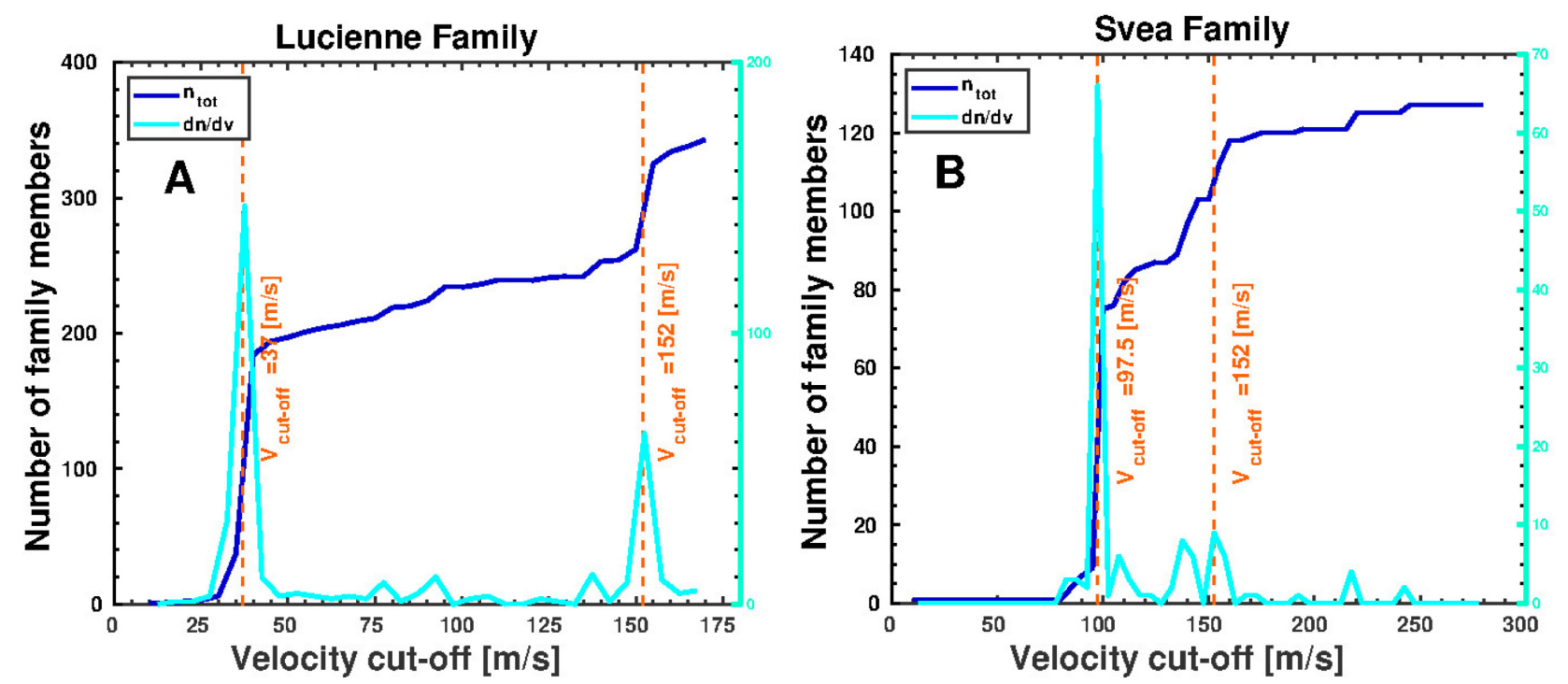

Figure 9. The number of asteroid members of the Lucienne (panel A) and Svea (panel B) families as a function of the cut-off velocity. The blue and cyan lines are the cumulative and differential distributions, respectively. The vertical dashed lines indicate the cut-off limits to define the minimum and maximum number of members in each family.
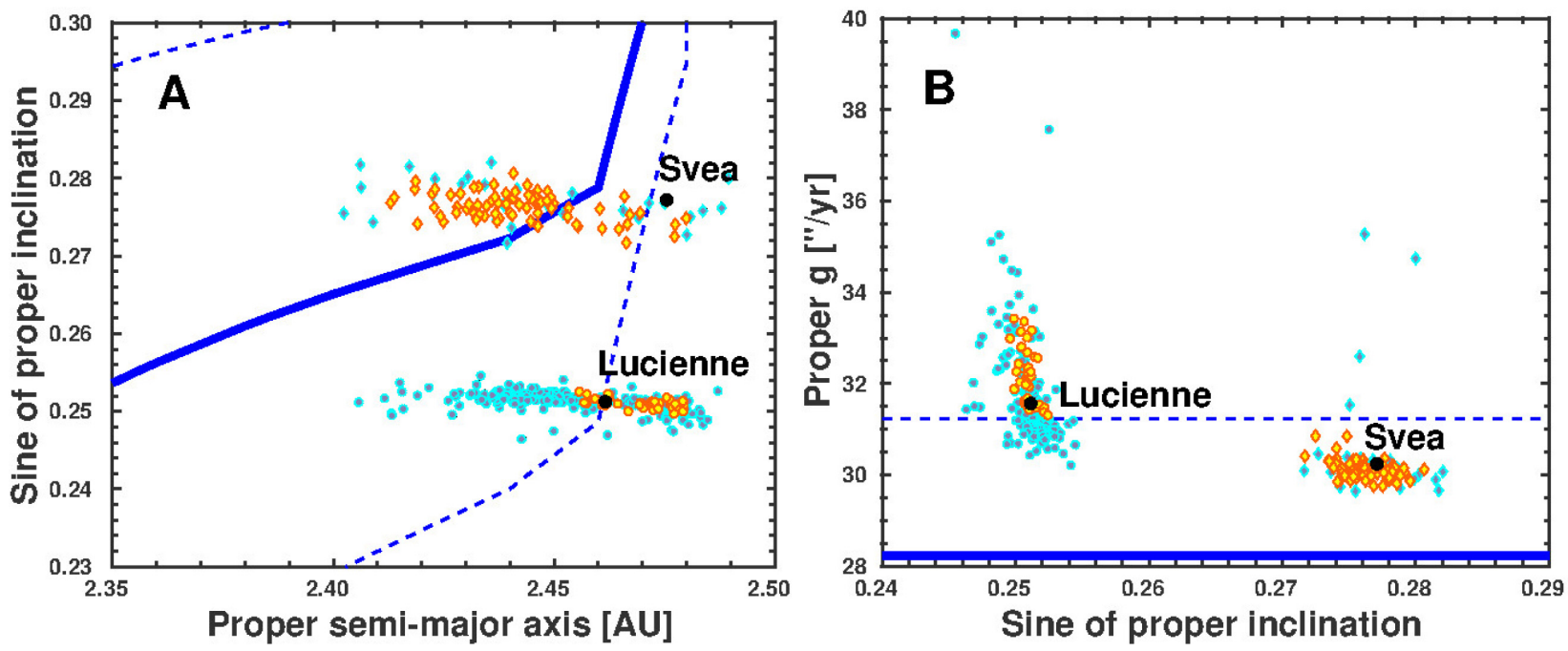

Figure 10. Distribution of the members of the Lucienne (circles) and Svea (diamonds) families in the space of proper elements and $g$ frequency (from the AstDys catalogue). The minimum number and maximum number of members are identified by the orange and cyan colours, respectively. The blue lines determine the centre (full) and approximate borders (dashed) of the $v_{6}$ resonance.

resonance with Jupiter. The orange and cyan colours identify the family members for the considered minimum and maximum values of $v_{\text {cut }}$, respectively. The full blue line corresponds to the locus of the $v_{6}$ resonance, computed using the analytical model of Milani \& Knežević (1994) for a fixed eccentricity of 0.1 [i.e. compatible with the proper eccentricities of asteroids (1892) Lucienne and (329) Svea]. The blue dotted lines approximately encompass the resonant domain.

As we can see, the Svea family is strongly influenced by the $v_{6}$ resonance. For the maximum cut-off, we find 56 members ( 54 per cent) of this family evolving in anti-aligned libration. For the Lucienne family, on the other hand, we find only one member evolving in an anti-aligned libration state. Interestingly, neither (1892) Lucienne nor (329) Svea is in a resonant state, having their critical arguments always circulating.

The same procedure described above has been applied to the other families and clumps presented in Fig. $8{ }^{3}$ We recover the results by Carruba \& Morbidelli (2011a) for the Tina family; we identified 86 family members ( 90 per cent) in anti-aligned libration. We also recover the results for the Euphrosyne family (Carruba

${ }^{3}$ Baptistina, Chimaera, Datura, Flora, Klio, Levin, Lucascavin, Massalia, Matterania, Phocaea, Sulamitis, Vesta, Emilkovalski, Gersuind, Hansa, Tina, Watsonia, 2000 DL37 Cindygraber, Euphrosyne, 1995 SP1, 2000 DQ94. 
Table 3. Asteroid families and clumps that interact with the $v_{6}$ secular resonance. The second and third columns give the minimum and maximum cut-off values considered to define the family, the fourth column gives the maximum number of members, and the fifth column indicates the number of members in anti-aligned libration.

\begin{tabular}{lcccc}
\hline Family name & $\begin{array}{c}\text { Min. } v_{\text {cut }} \\
\left(\mathrm{m} \mathrm{s}^{-1}\right)\end{array}$ & $\begin{array}{c}\text { Max. } v_{\text {cut }} \\
\left(\mathrm{m} \mathrm{s}^{-1}\right)\end{array}$ & Members & Librators \\
\hline (1892) Lucienne & 37 & 150 & 254 & 1 \\
(329) Svea & 97.5 & 150 & 103 & 56 \\
(1222) Tina & 200 & 260 & 96 & 86 \\
(31) Euphrosyne & 45 & 95 & 3408 & 20 \\
(7605) Cindygraber & 95 & 135 & 93 & 2 \\
(16708) 1995 SP1 & 32.5 & 45 & 331 & 4 \\
\hline
\end{tabular}

et al. 2014), with 20 members in anti-aligned libration. Finally, we identify in the outer main belt two clumps with few members also in anti-aligned libration. Our results are summarized in Table 3.

As stated in Section 3, the drawback of using the synthetic proper elements provided by AstDys is that they are not expected to be accurate enough for asteroids evolving inside the $v_{6}$ resonance. Therefore, in the case of asteroids in librating states, it would be necessary to substitute the standard $e_{\mathrm{p}}$ by a suitable resonant proper element $\Delta e_{\mathrm{p}}$ (e.g. Milani et al. 2017). This resonant proper eccentricity can be roughly computed, for example, as the amplitude $\left|e_{\max }-e_{\min }\right|$ between the minimum and maximum values of the filtered series of $e \cos \sigma_{6}, e \sin \sigma_{6}$. Nevertheless, this procedure would not help to improve the analysis of the families, because not all the members are purely resonant, and the proper elements $e_{\mathrm{p}}$ and $\Delta e_{\mathrm{p}}$ are not comparable to each other. Only in the case of the Tina family, which has most of its members inside the $v_{6}$ resonance, would this procedure be worth applying.

\section{CONCLUSIONS}

We identify and study the dynamical evolution of asteroids affected by the $v_{6}$ secular resonance in the main belt, with particular emphasis on those bodies that exhibit states of pure libration. Our results can be summarized as follows:

(i) From a simulation of the dynamical evolution over $20 \mathrm{Myr}$, we identify a population of 803 asteroids that are trapped in either aligned libration (resonant argument $\varpi-\varpi_{6}$ oscillating around $0^{\circ}$ ) or anti-aligned libration (resonant argument oscillating around $180^{\circ}$ ).

(ii) The population of asteroids in aligned libration is constituted by only seven objects, all located in the central main belt. This is the first time that such a kind of aligned librators have been detected. Our numerical simulations of their orbits over \pm 200 Myr show that only 4 of them remain in stable aligned libration $(e \sim 0.1)$ over the whole time-span, while the other three are also stable, but at some point they begin to alternate their resonant argument moving between phases of libration and circulation, with $e<0.3$.

(iii) The population of asteroids that are presently alternating between aligned libration and circulation is constituted by 21 bodies in the middle and outer main belt. More than half of these asteroids are unstable over a $\pm 200 \mathrm{Myr}$ time-span, being scattered to the outer belt and removed by planetary close encounters.

(iv) The largest depletion of the $v_{6}$ resonance occurs in the regions where the analytical model of Yoshikawa (1987) predicts that asteroids should be found in aligned libration states. This would be the reason why the population of such bodies is presently too small.

(v) We identified two new asteroid families, Svea and Lucienne, both in the inner main belt, and two new clumps, Cyndygraber and 1995 SP1, in the outer main belt, which have members that interact with the $v_{6}$ resonance in anti-aligned libration states. In particular, the Svea family has the second largest population of resonant members, following the family of Tina (Carruba \& Morbidelli 2011a).

Our results indicate that the $v_{6}$ secular resonance is a very effective mechanism of depletion throughout the main asteroid belt. The resonance affects the presently known asteroid families very little, with a few exceptions, basically because any large family fully formed in the resonant domain would have been quickly depleted/dispersed and is no longer there to be detected. The only exceptions are the small families of Svea, Tina, and a group of asteroids in aligned libration that are inside the resonance but can be stable for hundreds of Myr. We conclude that these asteroids and families are particularly stable because they do not interact with other dynamical mechanisms, such as mean motion and other linear and non-linear secular resonances. A study of their stability over longer time-scales would require taking into account the Yarkovsky and YORP effects, but that is beyond the scope of this work. In any case, such studies would help to set tight constraints to the ages of these small families.

\section{ACKNOWLEDGEMENTS}

We are grateful to the reviewer of this paper, Prof. Andrea Milani, for his comments and suggestions that significantly improved the quality of this work. We also wish to thank the Brazilian National Research Council (CNPq) that supported this work via the grants 300733/2017-8, 305453/2011-4, 310317/20169, the São Paulo State Science Foundation (FAPESP) grant 16/04476-8, and the Rio de Janeiro State Science Foundation (FAPERJ). We are also grateful to the Mathematics Department of UNESP, campus of Guaratinguetá, for the use of their facilities. Part of the simulations have been performed at the SDumont Supercomputer of the Brazilian National System of HPC (SINAPAD).

\section{REFERENCES}

Brouwer D., van Woerkom A. J. J., 1950, Astron. Papers Amer. Eph. and Naut. Alm., 13, 81

Brož M., 1999, Master's thesis. Charles University, Prague

Carruba V., 2009, MNRAS, 395, 358

Carruba V., 2010, MNRAS, 408, 580

Carruba V., Morbidelli A., 2011a, MNRAS, 412, 2040

Carruba V., Machuca J., 2011b, MNRAS, 418, 1102

Carruba V., Aljbaae S., Souami D., 2014, ApJ, 792, 46

Carruba V., Nesvorný D., Vokrouhlický D., 2016, AJ, 151, 164

Charlier C. V. L., 1902, Die Mechanik des Himmels. Verlag Von Veit \& Comp., Leipzig, 410

Froeschlé C., Scholl H., 1993, Cel. Mech. Dyn. Astron., 56, 163

Froeschlé C., Hahn G., Gonczi R., Morbidelli A., Farinella P., 1995, Icarus, 117,45

Gil-Hutton R., 2006, Icarus, 183, 93

Hagihara Y., 1928 , Proc. of the Physico-Mathematical , Soc. of Japan, 3rd series, Vol 10., 127

Knežević Z., Milani A., 2003, A\&A, 403, 1165

Knežević Z., Milani A., Farinella P., Froeschlé C., Froeschlé C., 1991, Icarus, 93,316 
Levison H. F., Duncan M. J., 1994, Icarus, 108, 18

Machuca J. F., Carruba V., 2012, MNRAS, 420, 1779

Milani A., Knežević Z., 1990, Cel. Mech. Dyn. Astron, 49, 347

Milani A., Knežević Z., 1994, Icarus, 107, 219

Milani A., Cellino A., Knežević Z., Novaković B., Spoto F., Paolicchi P., 2014, Icarus, 239, 46

Milani A., Spoto F., Knežević Z., Novaković B., Tsirvoulis G., 2016, in Chesley, S. R. and Morbidelli A, Jedicke, R. and Farnocchia, D., eds, Proc. IAU Symp. Vol. 318, Asteroids: New Observations, New Models., 28

Milani A., Knežević Z., Spoto F., Cellino A., Novaković B., Tsirvoulis G., 2017, Icarus, 288, 240

Morbidelli A., 1993, Icarus, 105, 48

Morbidelli A., Henrard J., 1991a, Cel. Mech. Dyn. Astron, 51, 131

Morbidelli A., Henrard J., 1991b, Cel. Mech. Dyn. Astron, 51, 169

Mothe-Diniz T., Roig F., Carvano J. M., 2005, Icarus, 174, 54
Nesvorný D., Brož M., Carruba V., 2015, in Michel P., DeMeo F. E., Bottke W. F., eds, Identification and Dynamical Properties of Asteroid Families, Asteroids IV, Univ. Arizona Press and LPI, Tucson, AZ, 297

Nobili A. M., Milani A., Carpino M., 1989, A\&A, 210, 313

Sidlichovský M., Nervorný D., 1996, Cel. Mech. Dyn. Astron, 65, 137

Spoto F., Milani A., Knežević Z., 2015, Icarus, 257, 275

Tisserand F., 1882, Annales de l'Observatoire de Paris, 16, E.1

Williams J. G., 1969, PhD thesis. University of California, Los Angeles

Williams J. G., Faulkner J., 1981, Icarus, 46, 390

Yoshikawa M., 1987, Celestial Mechanics, 40, 233

Zappalà V., Cellino A., Farinella P., Knežević Z., 1990, AJ, 100, 2030

This paper has been typeset from a $\mathrm{T}_{\mathrm{E}} \mathrm{X} / \mathrm{LET}_{\mathrm{E}} \mathrm{X}$ file prepared by the author. 